\title{
Qualitätsmanagement
}

\section{Neue Regeln und Aufgaben für alle Ärzte}

\author{
Für Vertragsarztpraxen, Kliniken und Zahnärzte gilt künftig eine \\ gemeinsame Richtlinie, was das Qualitätsmanagement anbelangt. \\ Die neuen Regeln bringen Ärzten in Praxen mehr Pflichten.
}

$\mathrm{N}$ och ist die neue Richtlinie zum Qualitätsmanagement (QM) zwar nicht in Kraft, doch die Erfahrung aus den vorangegangenen Richtlinien, die der Gemeinsame Bundesausschuss (GBA) - damals noch für alle drei Versorgungssektoren einzeln - beschlossen hat, zeigt, dass sich Vertragsärzte besser schon einmal auf die veränderten Anforderungen an ihr Praxis-QM einstellen sollten. An der „Erstfassung“, wie sie der GBA selbst nennt und die der Ausschuss am 17. Dezember 2015 abgesegnet hat, dürfte nicht mehr viel nachgebessert werden.

Mit der Einheits-Richtlinie für Vertragsärzte, Vertragszahnärzte und Kliniken soll vor allem die Qualität in der sektorübergreifenden Versorgung transparenter und besser werden. Dazu wird als neues QM-Instrument etwa ein Schnittstellenmanagement eingeführt. Dieses soll die gezielte Kommunikation und abgestimmte Prozesse aller Beteiligten beinhalten. Dabei sollen die Übergänge entlang der Versorgungskette so gestaltet werden, „dass alle erforderlichen Informationen zeitnah zur Verfügung stehen und eine koordinierte Versorgung gewährleistet ist", heißt es in Paragraf 4 der neuen QM-Richtlinie. Konkreter äußert sich der GBA allerdings nicht. Das heißt im Endeffekt, dass die Praxen Ablaufbeschreibungen oder Checklisten für diese Schnittstellenbereiche erarbeiten sollten.

$\mathrm{Zu}$ dieser Änderung passt auch, dass in größeren Praxiseinheiten, in denen mehr als ein Arzt beschäftigt ist, künftig ein verantwortlicher QM-Arzt benannt werden muss - zusätzlich zur QM-Beauftragten, die aus dem Kreis der Praxismitarbeiterinnen stammen kann.

\section{Patientenaufklärung als eigene QM-Maßnahme}

Neu ist für Vertragsärzte zudem, dass die Patienteninformation und -aufklärung als eigene QM-Maßnahme angeführt wird. Hier sollen die Einrichtungen für den ,individuellen Arzt-Patienten-Kontakt" verständliche Informationen und Angebote von Selbsthilfeorganisationen und Beratungsstellen zusammenfassen. Ebenfalls Pflicht wird ein System für die Arzneimitteltherapiesicherheit sowie ein spezielles Management für Schmerzpatienten. Abweichen oder Verzichten auf eine der Pflichtmaßnahmen beziehungsweise eines der Pflichtinstrumente dürfen Ärzte nur, wenn sie dies durch besondere einrichtungsbezogene Rahmenbedingungen begründen können.

Beschwerde- und Fehlermanagement werden in der neuen Richtlinie zudem konsequent getrennt. Bereits mit der alten Richtlinie mussten Praxen beides haben. Das Fehlermeldesystem zielt jedoch eher auf eigene Mitarbeiter ab. Diese sollen "sanktionsfrei“ und anonym die Möglichkeit haben, Fehler und Beinaheereignisse $\mathrm{zu}$ melden, aus denen dann konkrete Maßnahmen abgeleitet werden.

Neu ist auch, dass neben der regelmäßigen Patientenbefragung eine regelmäBige Mitarbeiterbefragung stattzufinden hat. Hier stellt sich allerdings die Frage, ob in kleinen Praxiseinheiten - auf- grund der kaum möglichen Anonymität - nicht doch auf diese Maßnahme verzichtet werden kann.

\section{Neue Methodik zur Evaluierung erarbeitet}

Und noch etwas ändert sich: Der GBA habe Regelungen getroffen, mit denen die Einführung und Weiterentwicklung des Qualitätsmanagements „zukünftig systematisch evaluiert und veröffentlicht“ werde, sagt Dr. Regina Klakow-Franck, Vorsitzende des Unterausschusses Qualitätssicherung im GBA. „Das Institut für Qualitätssicherung und Transparenz im Gesundheitswesen (IQTIG) wird hierzu eine neue Methodik erarbeiten“, ergänzt Klakow-Franck. Damit wird auch für die Vertragsärzte künftig das IQTiG das Prüfverfahren vorgeben.

Der GBA hat jedoch erkannt, dass bei den Vertragsärzten das QM seit Jahren bereits gut läuft. Die bislang jährlichen Stichprobenprüfungen unter den Praxen werden nun nur noch zweijährig durchgeführt. Für 2015 gab es daher keine Stichprobe, die nächste ist für dieses Jahr angedacht. Hierbei bleibt die Maßgabe erhalten: QM-Muffeln droht zunächst nur eine Beratung durch ihre KV, Sanktionen gibt es auch künftig nicht. Allerdings gilt die Zweijahresregelung nur solange, bis das IQTiG besagte neue Kriterien zur Erhebung des QM-Standes nach der neuen sektorübergreifenden Richtlinie erarbeitet hat.

Für die Vertragsärzte wurde zudem die Frist für die Einführung und Umsetzung des QM gekürzt. Haben sie dafür nach der alten Richtlinie insgesamt fünf Jahre Zeit (je zwei Jahre für Einführung und Umsetzung sowie ein weiteres Jahr für die Selbstbewertungs- und Weiterentwicklungsphase), sind es nach der neuen Richtlinie nur noch drei Jahre.

Rebekka Höhl 